\title{
Proline-Rich Domain
}

National Cancer Institute

\section{Source}

National Cancer Institute. Proline-Rich Domain. NCI Thesaurus. Code C14106.

Proline-Rich Domains may be involved in heterotypic protein-protein interactions in signal transduction or other pathways. Found in signaling and structural proteins, WW domains are composed of approximately 40 amino acids folded as a stable, triple stranded betasheet that recognize proline-rich sequences. Some WW domains show a remarkable similarity to SH3 domains, which also appear to recognize some proline-rich sequences. Ena/VASP (Drosophila Enabled/Vasodilator-Stimulated Phosphoprotein) Family members regulate actin filament assembly, often through association with binding partners that display a proline-rich FPPPP motif. Ena/VASP proteins interact with these partners via the highly conserved Ena/VASP homology 1 (EVH1) domain. (NCI) 